\title{
Attention of Cancer Care for Totally Implantable Venous-access Ports Associate Complications the Latest 6 Years Analysis of 400 Cancer Chemotherapy Patients
}

\author{
Hiroshi Osawa $^{1^{*}}$, Yoshie Takazawa ${ }^{2}$, Tamayo Kameoka ${ }^{2}$, Ryoko Yanai ${ }^{2}$, Chie Hirosawa ${ }^{2}$ \\ ${ }^{1}$ Department of Oncology and Hematology, Edogawa Hospital, Tokyo, Japan \\ ${ }^{2}$ Department of Nursing, Edogawa Hospital, Tokyo, Japan
}

\section{Abstract}

Background: Regardless of the fact that we often perform totally implantable venous-access ports (TIVAPs) for cancer chemotherapy, there have been several reports regarding complications of its use in the world. The objective of this study was to summarize the TIVAPs idiopathic complications of 400 cancer chemotherapy patients in latest 6 years. The patients underwent TIVAPs in the internal jugular or subclavian vein via the seldinger techniqueunder fluoroscopic control by surgeon. We used two different devices, which were the Bard X-Port ${ }^{\mathrm{Tw}}$, used from 2009 to 2012, and the Power Port ${ }^{\mathrm{Tw}}$ used from 2012 to present. These devices are composed of titanium and silicone rubber port (DomePort ${ }^{\mathrm{m}}$, Bard Inc., Salt Lake City, UT, USA) connected to an 8 Fr silasticGroshong ${ }^{\text {Thx }}$ catheter tube.

Results: Four hundred fourdevices in 400 patients, a total of 121,856 days' insertions, with a median follow up of 388 days. There were 30 idiopathic complications. The total complication rate was $7.5 \%$ (30/400). The early complication rate was $0.75 \%$ (3/400) which reason was pneumothorax under the seldinger technique even used ultrasonic machine. The late complications consisted of eleven complete occlusions $(2.75 \%)$, eight pocket infections (2\%), three of pneumothorax $(0.75 \%)$, two pinch-off $(0.5 \%)$, two slip-off due to pedunculated breast $(0.5 \%)$, one catheter-related bacteremia $(0.25 \%)$, one wound dehiscence due to bevacizumab $^{\text {Tw }}(0.25 \%)$, one rubber port disconnection $(0.25 \%)$, and one rubber port rotation $(0.25 \%)$ in pocket. Especially, we experienced $12 \mathrm{~cm}$ length red thrombus and elevated D-dimer which possibility triggered Trousseau syndrome in parallel.

Conclusion: Although there were only 7.5\% (30/400) catheter-related complications with these TIVAPs, justifying its use, we have to be careful with complications such as a pinch-off, slip-off, infection, disconnection and occlusion.

\section{Introduction}

Totally implantable venous-access ports (TIVAPs)are useful for long-term nutrition management, intra-artery or intra-venous infusion of cancer chemotherapy, blood transfusion, and blood sampling. Recently, TIVAPs are improving the quality of life (QOL) of the patient for such reasons. Furthermore, TIVAPs is frequently used for colon cancer in oxaliplatin (L-OHP), irinotecan (CPT-11), and 5-fluorouracil (5FU) combination chemotherapy (FOLFOX, FOLFIRI) and pancreatic cancerfor FOLFIRINOX. However, there are several early and late complications due to TIVAPs insertion. The early complications are as follows; 1)pneumothorax, 2) hematothorax, 3) blood vessel damage, 4)air embolus. The late complications are as follows; 1) pinch-off, 2) fibrin sheaths and fibrin sleeve, 3) occlusion, 4) catheter damage, 5) infection. In addition, an infusion drug catheter indwelling position and use reason of the catheter and various TIVAPs troubles such as the condition of a patient of the patient and management of a patient's oneself, malfunction of degree of achievement and situation and port catheter in itself for the handling of a maneuver and the person of medical care of the management are reported [1]. Recently, there are several reports regarding the use of TIVAPs problems in Japan [2,3] and another countries [4,5]. We have been reported to single institutes the TIVAPs complications of 404devices in 400 patients, a total of 121,856 days' insertions, with a median follow up of 388 days in the latest 6 years.

\section{Patients and methods}

Aims: To investigate the early and late complications by totally implantable venous-access ports in single institute study research.
Patients: Patients who confirmed solid cancer and hematological malignancies, and who met the following inclusion criteria 1-6) were included. 1) Over20-year-old, 2) major organ function preserved (if $\mathrm{D}$-dimer $>1.0 \mu \mathrm{g} / \mathrm{ml}$, we have checked deep vein thrombosis of lower extremities by ultrasonography), 3) Eastern clinical oncology group (ECOG) performance status (PS): 0-2, 4) Confirmed solid tumor or hematological malignancies as a histologically, 5) no serious complications (any infections, Fever, diarrhea, thrombus,etc.),6) provided written consent based on informed consent. This study was carried out according to the regulation of local ethics committee of our hospital and according to the Declaration of Helsinki.

Methods: All TIVAPs inserted in the internal jugular or subclavian veins in patients were at our institute from January 2009 to December 2014. The optimal purpose of these TIVAPs was to fulfill requirements for long term chemotherapy or hyper alimentation. The operations on these patients were under gone by our institutes' surgeon, who chose the insertion position according to patients' condition. The patients underwent to TIVAPs in internal jugular or subclavian vein via the

${ }^{\circ}$ Corresponding Author: Dr. Hiroshi Osawa, Department of Oncology and Hematology, Edogawa Hospital, 2-24-18 Higashi-koiwa, Edogawa-ku, Tokyo 133-0052, Japan; E-mail: oosawa@edogawa.or.jp

Citation: Osawa H, Takazawa Y, Kameoka T, YanaiR, Hirosawa C (2016) Availability of Thickness Estimation of the Subcutaneous Fat by Using the Nearinfrared Ray Measuring Device. Int J Nurs Clin Pract 3: 210. doi: https://doi. org/10.15344/2394-4978/2016/211

Copyright: (c) 2016 Osawa et al. This is an open-access article distributed under the terms of the Creative Commons Attribution License, which permits unrestricted use, distribution, and reproduction in any medium, provided the original author and source are credited. 
Citation: Osawa H, Takazawa Y, Kameoka T, YanaiR, Hirosawa C (2016) Availability of Thickness Estimation of the Subcutaneous Fat by Using the Near-infrared Ray Measuring Device. Int J Nurs Clin Pract 3: 210. doi: https://doi.org/10.15344/2394-4978/2016/211

Page 2 of 6

seldinger technique under fluoroscopic control. We used two different devices, which were the Bard X-Port ${ }^{\mathrm{is}}$, used from 2009 to 2012 and the Power Port ${ }^{\mathrm{sm}}$ from 2012 to present. These devices are composed oftitanium and silicone rubber port (DomePort ${ }^{\mathrm{ma}}$, Bard Inc., Salt Lake City, UT, USA) connected to an $8 \mathrm{Fr}$ silasticGroshong ${ }^{\text {Tix }}$ catheter tube. When they finished insertions of the TIVAPs, the device was filled with a saline solution. If a patient received bevacizumab ${ }^{\text {mo }}(\mathrm{Bmab})$ combined chemotherapy, TIVAPsuse was avoided for at least four weeks.

Evaluation: We defined early complications, which occurred within 30 days, and late complications occurred over 30 days. If any kind of TIVAPs-related complication was suspected, we always discussed the matter witha surgeon, radiologists, cardiologists and infection control team. In the case ofa catheter fever, we performed a blood culture using peripheral blood and port-catheter blood. In the case ofa catheteroc clusion, we performed pulping flash at first or removed the catheter in the end. In the case of a catheter pinch-off, chest X-rays and sagittal and coronal computed tomography were taken.

Observation period: The patients enrolled from January 2009 to December 2014. The end of observation period was January $1^{\text {st }} 2015$.

\section{Results}

\section{Patients Characteristics}

We showed patient characteristics (Table 1). Four hundred fourdevices in 400 patients were inserted at our hospital from 2009 to 2014. Two hundred thirty-seven patients were male (59\%) and one hundred sixty-three patients were female (41\%). The median age was 62-years old (range: 31-84years). These devices, a total of 121,856 days in situ, were inserted with a median follow up of 388 days (range: 31 2035 days). The primary reason for TIVAPs was chemotherapy for cancer chemotherapy. These populations were three hundred thirtyseven solid cancers and sixty-three hematological malignancies. The inserted location of TIVAPs were more subclavian vein than jugular vein, but we have changed the insertion approach from subclavian to jugular vein, because there is the risk of getting TIVAPs caught between the first rib and clavicles. There were three early complications of pneumothorax $(0.75 \%)$. The late complications consisted of eleven complete occlusions (2.75\%), eight pocket infection (2\%), two pinch-off $(0.5 \%)$, two slip-off due to pedunculated breast $(0.5 \%)$, one catheter-related bacteremia $(0.25 \%)$, one wound dehiscence due to Bmab $(0.25 \%)$, one rubber port disconnection $(0.25 \%)$, and one rubber port rotation $(0.25 \%)$ in pocket.We have not shown available curve. We defined the event case which caused TIVAPs related complications. On the contrary, the censored case ended the chemotherapy or death. The accumulation availabilityrate was $95 \%$ in one year, two years $81.9 \%$, and three years $74.4 \%$. We showed to pay attention of late complication cases.

\section{1) Early complication cases}

\section{a) Pneumothorax}

There were only $0.75 \%$ (3/400) of pneumothorax as an early complications. The inserted location of TIVAPs were more subclavian vein than jugular vein. These catheters were inserted by surgeon.

\section{2) Late complication cases}

a) Catheter pinch-off (Case 1)

\begin{tabular}{|c|c|c|}
\hline & & No. of Patients \\
\hline Gender & $\begin{array}{l}\text { Male } \\
\text { Female }\end{array}$ & $\begin{array}{l}237(59 \%) \\
163(41 \%)\end{array}$ \\
\hline Age & $\begin{array}{l}\text { Median } \\
\text { Range }\end{array}$ & $\begin{array}{l}62 \text { years } \\
31-84 \text { years }\end{array}$ \\
\hline No. of TIVAPs & $\begin{array}{l}2009 \\
2010 \\
2011 \\
2012 \\
2013 \\
2014\end{array}$ & $\begin{array}{l}90(23 \%) \\
64(16 \%) \\
52(13 \%) \\
69(17 \%) \\
56(14 \%) \\
69(17 \%)\end{array}$ \\
\hline Access route & $\begin{array}{l}\text { Right subclavian } \\
\text { Light subclavian } \\
\text { Right internal jugular } \\
\text { Light internal jugular } \\
\text { Femoral }\end{array}$ & $\begin{array}{l}150(37 \%) \\
102(25 \%) \\
91(23 \%) \\
54(14 \%) \\
3(1 \%)\end{array}$ \\
\hline $\begin{array}{l}\text { Solid cancer } \\
\text { Hematological } \\
\text { malignancies }\end{array}$ & $\begin{array}{l}\text { Colorectal } \\
\text { Gastric } \\
\text { Esophageal } \\
\text { Pancreas } \\
\text { Lung } \\
\text { Breast } \\
\text { Head \& Neck } \\
\text { Lymphoma } \\
\text { Myeloma } \\
\text { Myelodysplastic syndrome } \\
\text { Leukemia }\end{array}$ & $\begin{array}{l}134(33 \%) \\
80(20 \%) \\
53(13 \%) \\
35(9 \%) \\
23(8 \%) \\
9(2 \%) \\
3(1 \%) \\
28(7 \%) \\
19(4 \%) \\
13(3 \%) \\
3(1 \%) \\
\end{array}$ \\
\hline $\begin{array}{l}\text { Early Complications } \\
\text { Late Complications }\end{array}$ & $\begin{array}{l}\text { Pneumothorax } \\
\text { Occlusion } \\
\text { Pocket infection } \\
\text { Pinch-off } \\
\text { Slip-off } \\
\text { Bacteremia (MRSA) } \\
\text { Disconnection } \\
\text { Wound dehiscence } \\
\text { Rubber port rotation }\end{array}$ & $\begin{array}{l}3(0.75 \%) \\
11(2.75 \%) \\
8(2 \%) \\
2(0.5 \%) \\
2(0.5 \%) \\
1(0.25 \%) \\
1(0.25 \%) \\
1(0.25 \%) \\
1(0.25 \%)\end{array}$ \\
\hline
\end{tabular}

Table 1: Patients Characteristics.

They were 62-year-old man who had advanced colon cancer. Since hiscatheter of case 1 in 12 months could not inject any solutions, we took achest X-ray (Figure 1a), changed contrast X-ray (Figure 1b) and computed tomography scan (CT). These images showed the fall of his catheter into the pulmonary artery (Figure 1c). We inserted 14 French (Fr.) $45 \mathrm{~cm}$ sheath from a right femoral vein. I derived a catheter in pulmonary artery with the Burman catheter with the tip aperture and derived 4Fr. multi-purpose catheter in pulmonary artery with a 0.025 guide wire and derived Hear trail IIJR4.0 in pulmonary artery with a $0.035 \mathrm{~mm}$ spring wire. A tip got into the catheter which I deviated from. I held the catheter which I got into in right pulmonary artery with a snare and I drew it out and removed it outside a sheath. I removed a sheath and pressed it and stopped bleeding, confirmed it, and was finished. We made sure the bent mark of the catheter and weak pressure marks ofthe catheter lock (Figure 1d).

\section{b) Disconnection (Case 2)}

She was a 62 -year-old womanwho had advanced colon cancer. When case 3 catheter in 24 months could not inject anti-emesis drug prior chemotherapy, she felt pain and her cutaneous color had gradually turned red (Figure 2a). As soon as we imaged chest X-rays (Figure $2 \mathrm{~b})$, which showed the rubber port disconnect between port and catheter (Figure 3c), we removed this catheter immediately. 
Citation: Osawa H, Takazawa Y, Kameoka T, YanaiR, Hirosawa C (2016) Availability of Thickness Estimation of the Subcutaneous Fat by Using the Near-infrared Ray Measuring Device. Int J Nurs Clin Pract 3: 210. doi: https://doi.org/10.15344/2394-4978/2016/211
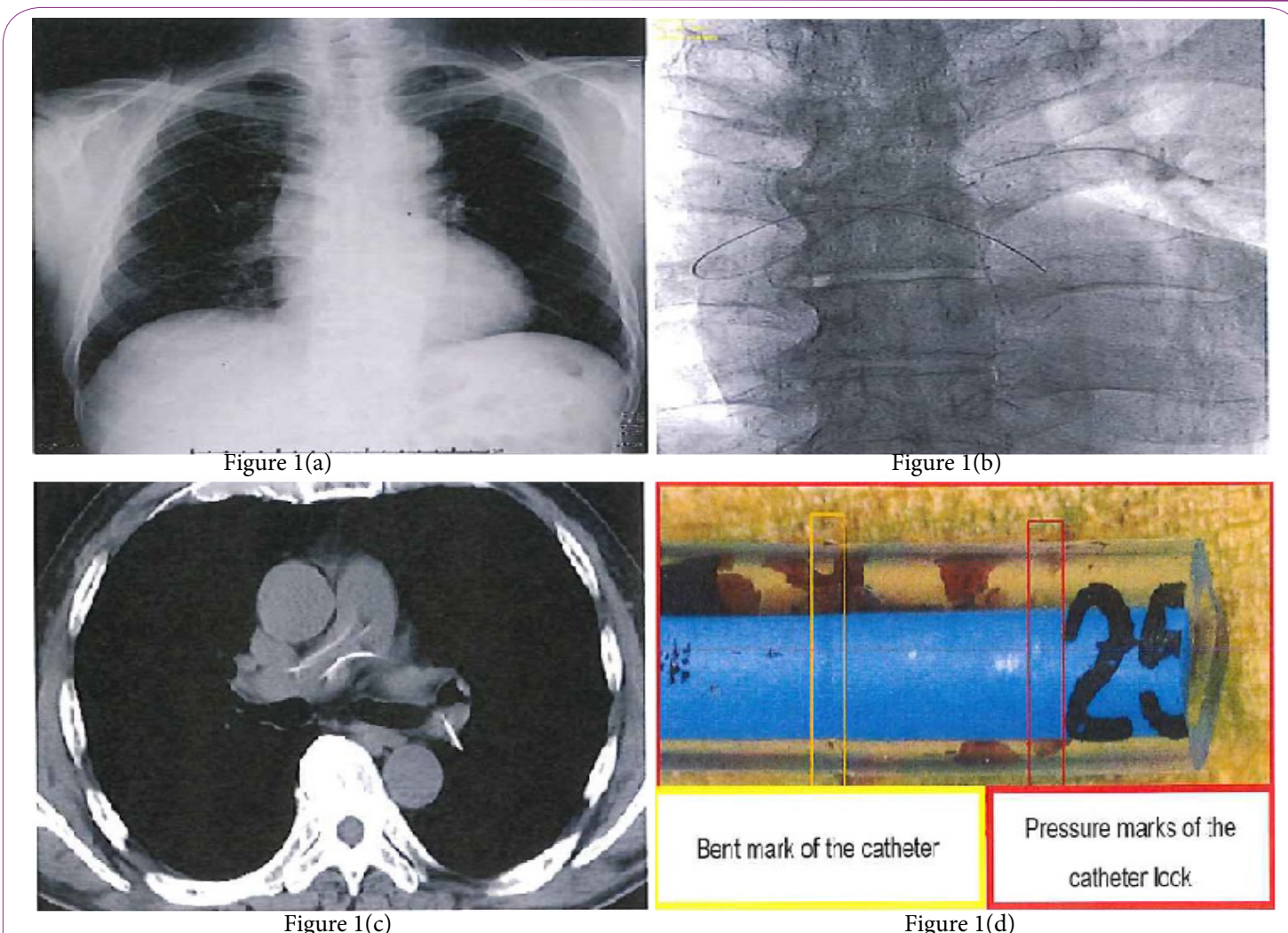

Figure 1(d)

Figure 1:

a) Missing the catheter tube by chest X-ray.

b) Pinch-off the catheter into pulmonary artery by changed contrast of chest X-ray.

c) Pinch-off the catheter into pulmonary artery by CT scan.

d) Bent mark of the catheter (yellow square) and weak pressure marks of the catheter lock (red square).

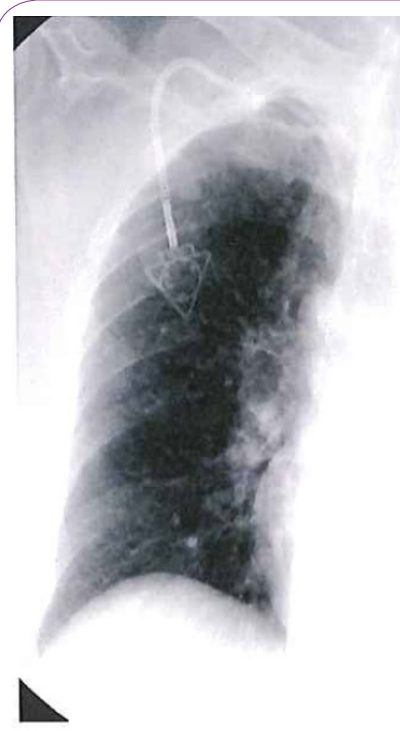

Figure 2(a)

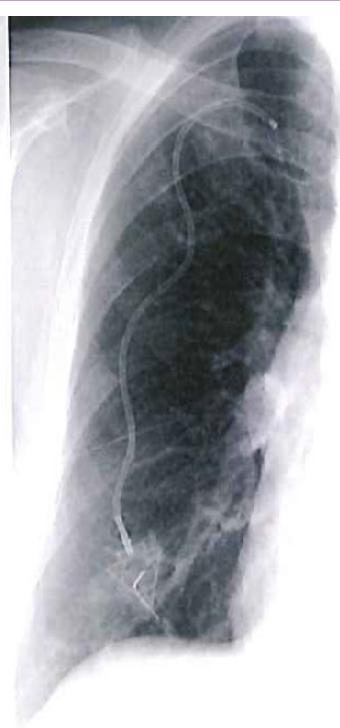

Figure 2(b)

Figure 2

a) Normal position of the catheter.

b) Slip-off from central venous in 7 months later.

c) Catheter slip-off (Case 3)

There was a 68-year-old woman (Figure $3 a$ ) who had advanced colon cancer. The catheter of case 2 in 7 months could not inject any infusion solution. We took a chest X-ray, which showed the catheter slip-off

from central venous (Figure $3 b$ ). We thought that these were due to pedunculated breast because her catheter was pulled towards the direction of gravity and a catheter had slipped off.

d) Complete occlusion (Case 4)

A 63-year-old man, who had a swallow disturbance, was diagnosed as having inoperable esophageal cancer. Even though,his TIVAP was always maintained by pulsing flash and continuous hyperalimentation,which catheter of case 4 in 4 months could not inject any infusion solution. We imaged chest X-rays (Figure 4a). Although we could not find a pinch-off and port disconnection, we took the catheter off immediately. We found complete occlusion of $12 \mathrm{~cm}$ length red thrombus (Figure 4b) in pathologically (data not shown). Additionally, the patient felt a speech disturbance and gait disturbance who had brain infarction (Figure 4c). Trousseau syndrome caused complete occlusion which revealed his D-dimer elevating gradually (Figure 4d).

\section{Discussion}

The aim of totally implantable venous-access ports (TIVAPs) had been for nutrition at home medicalcare and intra-arterial injection. Recently, the aims of TIVAPs haveadvancedto use for cancer chemotherapy. TIVAPs are frequently used for colon cancer in FOLFOX or FOLFIRI, and pancreatic cancer in FOLFIRINOX for continuous intra-venous chemotherapy. Otherwise, the aims of TIVAPs were used not only incontinuous intra-venous chemotherapy, but also for securing vascular access because cancer patients are able 
Citation: Osawa H, Takazawa Y, Kameoka T, YanaiR, Hirosawa C (2016) Availability of Thickness Estimation of the Subcutaneous Fat by Using the Near-infrared Ray Measuring Device. Int J Nurs Clin Pract 3: 210. doi: https://doi.org/10.15344/2394-4978/2016/211

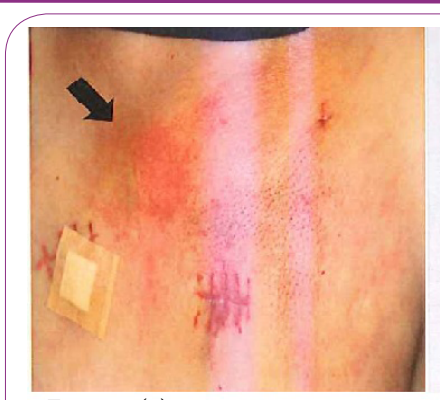

Figure 3(a)

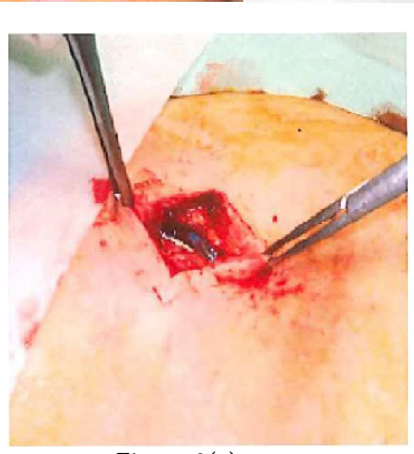

Figure 3(c)

Figure 3

a) The patients' cutaneous color had gradually turned red(black arrow) after chemotherapy

b) Rubber port disconnect between port and catheterby expanded chest X-ray

c)Rubber port disconnect between port and catheter under operation. to prolong their survivaltime as a result ofthe progress of chemotherapy and development of the molecular target drug.Of course, we have been them using for nutrition at home medical care too. TIVAPs are very important for a cancer patient's quality of life. We used two different devices, which were the Bard X-Port ${ }^{\mathrm{m}}$, used from 2009 to 2012, and the Power Port ${ }^{\text {tix }}$ from 2012 to present. These devices are composed of titanium and silicone rubber port connected to an $8 \mathrm{Fr}$ silasticGroshong $^{\text {Tix }}$ catheter tube. When they finished insertions of TIVAPs, the device was filled with a saline solution. At first, we discussed early complications concerning the catheter insertion. When we inserted TIVAPs, a catheter to subclavian vein, we experienced three cases of pneumothorax. We thought about this complication which concluded the position of the catheter insertiondepends on patient's condition and anatomy. We changed the insertion to the subclavian or the internal jugular vein. Next, we discussed late complications of the catheter insertion. However, we experienced two pinch-off and one rubber port disconnectionwhen they inserted a catheter to the subclavian vein. We thought the reason that pinch-off and disconnection reasons were the following; 1 ) the fixation of the stem part is insufficient, 2) the fixation of the catheter part is insufficient, 3) catheter was put between first rib and clavicles, 4) high pressure of pulsing flash in natural saline. Actually, when a catheter lock was put on appropriately, we recognized the characteristicof a wide pressure trace. However, we were not able to confirm a wide pressure trace. When we had changed insertion position from the subclavian to the internal jugular vein in 2012, we had not experienced apinch-off so far. We concluded that the major reasonfor pinch-offwas caused by the compression of a catheter between the first rib and clavicles.

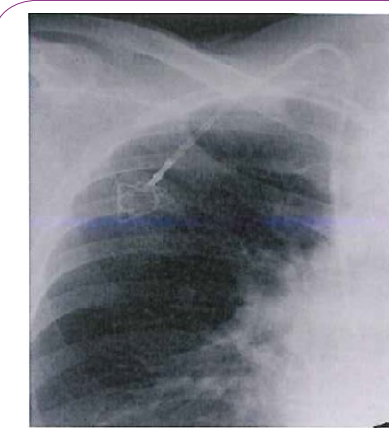

Fugure 4(a)

D-dimar $\mu \mathrm{g} / \mathrm{m}]$

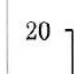

15

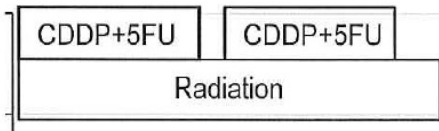

CDDP: $70 \mathrm{mg} / \mathrm{m}^{2}$, day1 \& $29,2 \mathrm{hr}$, div

$5 \mathrm{FU}: 700 \mathrm{mg} / \mathrm{m}^{2}$, day $1-4,29-32$, civ

XRT : $50.4 \mathrm{~Gy} / 28 \mathrm{Fr}$,

short-T area included supraclavicular lymph nodes

5

$$
6 / 2
$$

$6 / 21$

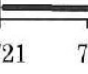

Figure 4

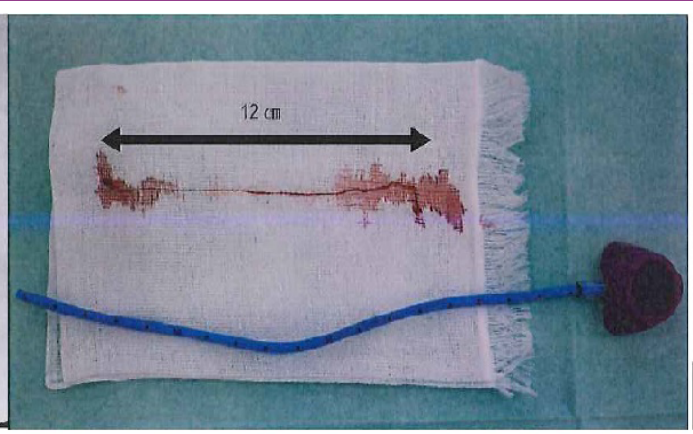

Fugure 4(b)

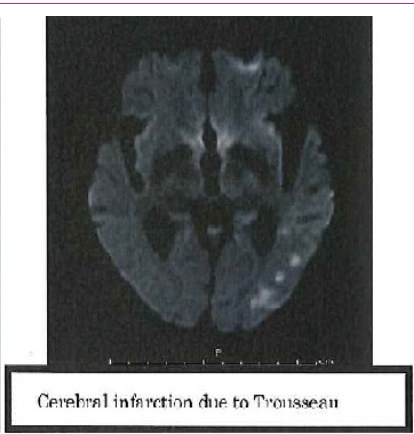

Fugure 4(c)

a) Normal position of the catheter

b) The complete occlusion of $12 \mathrm{~cm}$ length red thrombus in pathologically

c) The showered embolus by diffusion image of brain MRI

d) Onset and course of the patient received chemo-radiotherapy and movement of D-dimer 
Citation: Osawa H, Takazawa Y, Kameoka T, YanaiR, Hirosawa C (2016) Availability of Thickness Estimation of the Subcutaneous Fat by Using the Near-infrared Ray Measuring Device. Int J Nurs Clin Pract 3: 210. doi: https://doi.org/10.15344/2394-4978/2016/211

Page 5 of 6

It is observed in $1.1-5.0 \%$ of cases [6-8]. The complication of resistance causes myocardial or intravascular perforation, arrhythmia, endocarditis, pulmonary artery thrombosis or embolization and sepsis. We are better to be careful for pinch-off signs, which are infection, infra-clavicular discomfort, chest discomfort and swellingduring infusion any solutions $[9,10]$. Chenga reported that their removal ratio of catheter was $98 \%(90 / 92)$ by gooseneck catheter [11]. They could not remove resistances, which migrated and divided to peripheral side. If there is sign of catheter obstruction on radiography, remove catheter immediately to avoid more severe complications such as rupture and its migration 7). Slip-off of catheter due to pedunculated breast from central venous is not a rare report, but we need attention. When women of pedunculated breast are getting older, their breast position turns with a deceits and a standing position by aging. If a patient feels painful swelling of the clavicular area soon after flushing with natural saline, a chest X-ray must be taken. We experienced a similar situation with case 3 and 4 , whichhad pedunculated breast 3). Disconnection of the catheter from port is rare (0.1-2.0\%). However, we sometimes noticed that aless than $10 \mathrm{ml}$ syringe makes high pressure for pulsing flush, which increases the risk of rupture or disconnection $[6,12]$. If a patient feels painful swelling in the TIVAP insertion site and clavicular area soon after flushing with natural saline, a chest X-ray must be taken. We experienced with similar cases. The occlusion of catheter was easy to recognize, forinfusion and withdrawal of blood from the system was not possible. It occurs in $1.9-8.0 \%$ of cases, whose cause is clotted blood [9,12-14]. There wasa rare of $2.1 \%$ of complete occlusion in our institute.Kurul reported a catheter tip that rubs against the vein can produce a local thrombosis $[14,15]$. Nevertheless, precipitation from total parenteral nutrition, drugs, transfusion, or other intravenous solutions as well as particles that come from port membrane, port floor, or cutaneous tissue from punctures can obstruct the device $[7,12]$. Although we took a chest X-ray and computed tomography, we can carry out to exclude catheter malposition, kinking, disconnection, fracture and migration. And then, we pulled the catheter off as soon as possible, the thrombus of $12 \mathrm{~cm}$ was detected (Figure $5 \mathrm{~b}$ ). We required Baxter for this problem which could not detect any of the catheter's problems. Their comments are as follows; 1 ) there were not the malfunction of the backflow prevention valve of the catheter, the leak and the occlusion by an examination for function, 2) the cause that this thrombus produced was not clear without accepting transformation and the damage to a catheter in the investigation for appearance. Sakamato [3] reported that ifthe catheter outer diameter is thin, intravenous feeding speed of hyper-viscosity solution becomes slow. Otherwise, they improved the catheter's problems, which changed outer diameter from $6 \mathrm{Fr}$ to $8 \mathrm{Fr}$. In our case, it was not the catheter's outer diameter because our catheters were composed of an $8 \mathrm{Fr}$ catheter tube in Power Port $^{\text {tw }}$. We discussed this occlusion, whose reasons were luck of pulsing flash, poor performance status, poor nutrition, and long-term bed rest due to locally advanced esophageal cancer. And not only be using the catheter for chemo-radiotherapy, but also for hyper-alimentation.And we had already known Trousseau syndrome, which causesthrombosis or embolism in $1-11 \%$ of cancer patients $[16,17]$. Otherwise, we did not recognize anyhypercoagulabilityin blood sampling in this case. Finally, we concluded that this occlusion was occurred by several patients'conditions and catheters' directions.If cancer patients had poor performance status (PS3 or 4) in the ECOG, we do not recommend TIVAPs insertion.Infection is one of the most frequent complications of TIVAPs. Despite the use of all antiseptic barriers, a standardized surgical technique, not only were there four cases $(1.2 \%)$ of pocket infection, but one case also had bacteremia of Methicillin-resistant Staphylococcus aureus (MRSA). Although, TIVAPs is very useful equipment, we need to use aseptic technique and sterile barriers while manipulating catheters [18]. Theeight cases of pocket infections were controlled only with intravenous systemic antibiotics. Catheter removal was indicated in MRSA case because of bacteremia and catheter fever. Taniguchi reported that the aim of chemotherapy used TIVAPs were less than nutritious it. However, there is no evidence demonstrating any decrease in the infection rates [19]. Actually, there were a few catheter related infections in our institute, whose reasons are the use of catheter for cancer chemotherapy and we followed general maintenance and access procedures follow as; 1) wash hands, 2) wear clean gloves, 3 ) perform sterilization by povidone iodine, 4) puncture by needle and make sure metal sound, 5) 3-5 mL of blood and discard blood draws, 6) fix the needle under sterilization, 7) flush with $10-20 \mathrm{~mL}$ of normal saline by pulsing flush. Finally, the monoclonal antibodies are useful for solid censers and hematological malignancies. Especially Bmaband ramcirumnab ${ }^{\mathrm{Tm}}$ are an antiVEGF monoclonal antibodies which are central play of role cancer chemotherapy such as colon, lung, and breast. We experienced wound dehiscence of insertion of TIVAPs three months later in an advanced colon cancer patient who was treated by Bmab combined chemotherapy. We do not pay attention to only standardized Bmab complications such asthrombosis, hypertension and proteinuria, but also insertion of TIVAPs scar.Conclusions: Totally implantable venous-access ports are useful and safe for cancer patients who need to receive long time chemotherapy. We experienced early and late complications of TIVAPs, but there were low rates of major complications associated to TIVAPs system, justifying its use.

\section{Competing Interests}

The authors declare that they have no competing interests.

\section{References}

1. Kurul S, Saip P, Aydin T (2002) Totally implantable venous-access ports: local problems and extravasation injury. The Lancet OncolVol 3: 684-692.

2. Taniguchi K, Oka K, Tokuyasu N, Naka T, Kondo T, et al. (2008) Clinical outcome of totally implantable subcutaneous infusion port--comparison with home parenteral nutrition and chemotherapy. Gan To Kagaku Ryoho 35: $281-285$

3. Sakamoto E, Hasegawa H, Komatsu S, Norimizu S, Shingu Y, et al. (2013) [Complications after implantation of subcutaneous central venous port]. Gan To Kagaku Ryoho 40: 613-616.

4. Biffi R, de Braud F, Orsi F, Pozzi S, Mauri S, et al. (1999) Totally implantable central venous access ports for long-term chemotherapy. A prospective study analyzing complications and costs of 333 devices with a minimum follow-up of 180 days. Ann Oncol 9:767-773.

5. Nishihara K, Wolosker N, Bernardi CV,Yazbek G (2010) Totally implantable ports connected to valved catheters for chemotherapy: experience from 350 Groshong devices. J Vasc Access 11:17-22.

6. Lam AW, Chen YM, Yang KY, Tsai CM, Perng RP (1999) Disconnection of a venous Port-A-Cath followed by embolization after saline flush: rare case report. Jpn J Clin Oncol 29: 643-645.

7. Klotz HP, Schöpke W, Kohler A, Pestalozzi B, Largiadèr F (1996) Catheter fracture: a rare complication of totally implantable subclavian venous access devices. J Surg Oncol 62: 222-225.

8. Di Carlo, Fisichella P, Russello D, Puleo S, Latteri F (2000) Catheter fracture and cardiac migration: a rare complication of totally implantable subclavian venous access devices. J Surg Oncol 73: 172-173.

9. Koch HJ, Pietsch M, Krause U, Wilke H, Eigler FW (1998) Implantable venous access system: experience in 1500 patients with totally implanted central venous port system. World J Surg 22: 12-16. 
Citation: Osawa H, Takazawa Y, Kameoka T, YanaiR, Hirosawa C (2016) Availability of Thickness Estimation of the Subcutaneous Fat by Using the Near-infrared Ray Measuring Device. Int J Nurs Clin Pract 3: 210. doi: https://doi.org/10.15344/2394-4978/2016/211

10. Biffi R, De Braud F, Orsi F, Pozzi S, Arnaldi P, et al. (2001) A randomized, prospective trial of central venous ports connected to standard open-ended or Groshong catheters in adult oncology patients. Cancer 92: 1204-1212.

11. Chenga CC, Tasa TN, Yang CC, Han CL (2009) Percutaneous retrieval of dislodged totally implantable central venous access system in 92 cases: Experience in a single hospital. Eur J Radiol 69: 346-350.

12. Haindl H (1989) Technical complications of port-catheter systems. Reg Cancer Treat2:238-242.

13. Schwarz RE, Groeger J, Coit DG (1997) Subcutaneously implanted central venous access devices in cancer patients: a prospective analysis. Cancer 79: 1635-1650.

14. Viale PH, Yamamoto DS, Geyton JE (1999) Extravasation of infusate via implanted ports: two case studies. Clin J Oncol Nurs 3: 145-151.

15. Bow EJ, Kilpatrick G, Clinch JJ (1999) Totally implantable venous ports system for patients receiving chemotherapy for solid tissue malignancies: A randomized controlled clinical trial examining the safety, efficacy, costs, and impact on quality of life. J ClinOncol 17: 1267-1273.

16. Walsh-McMonagle D, Green D (1997) Low-molecular-weight heparin in the management of Trousseau's syndrome. Cancer 80: 649-655.

17. Uchiyama S (2008) Paraneoplastic neurological syndromes: Trousseau syndrome. Nihon Naika Gakkai Zasshi 10: 1805-1808.

18. Haire WD, Lieberman RP, Edney J, Vaughan WP, Kessinger A, et al. (1990) Hickman catheter-induced thoracic vein thrombosis. Frequency and longterm sequelae in patients receiving high-dose chemotherapy and marrow transplantation. Cancer 66: 900-908.

19. Yung B, Elborn JS, Campbell IA, Summers Y, Beckles M, et al. (1997) Thromboembolism related to a Port-a-Cath device in a patient with cystic fibrosis. Thorax 52: 98-99. 